\title{
BREVE HISTORIA DE LA BIBLIOTECA DE LA FACULTAD DE ENFERMERÍA DE LA UNIVERSIDAD PERUANA CAYETANO HEREDIA
}

\author{
EDITH CARMEN MUÑOZ
}

Resumen.- El artículo explora los inicios de la Biblioteca de la Facultad de Enfermería de la Universidad Peruana Cayetano Heredia con el fin de identificar sus aspectos más importantes. Se describirán sus antecedentes históricos vinculados al contexto institucional a partir de tres etapas significativas que ilustrarán el desarrollo de la biblioteca. La primera etapa, desde su origen en la Escuela de Enfermeras Arzobispo Loayza; la segunda etapa, con la creación como Facultad de Enfermería en el contexto universitario y, finalmente, la tercera etapa, que hace referencia a la situación actual.

Palabras claves: Historia; Bibliotecas de Enfermería; Escuelas de Enfermería; Bibliotecas Médicas; Biblioteca de la Facultad de Enfermería de la Universidad Peruana Cayetano Heredia.

\begin{abstract}
Summay.- This text explores the beginnings of the Library of the Faculty of Nursing at the Peruvian University Cayetano Heredia, in order to identify the most important aspects. There will be described his historical precedents linked to the institutional context from three significant stages that will illustrate the development of the Library. The first stage, from his origin in the School of Nursing Archbishop Loayza, the second stage, the creation like Nursing Faculty in the university context and finally the third stage, it refers to the current situation.
\end{abstract}

Key words: History; Libraries Nursing, Schools Nursing, Libraries Medical, The Nursing Library at Peruvian University Cayetano Heredia.

\section{Introducción}

Algunas veces, permanecemos tanto tiempo en una misma institución que podríamos desconocer su historia, pero para entregar una visión de lo que somos hoy y lo que queremos ser mañana debemos hacer una revisión cronológica para así descubrir cómo hemos ido cambiando, avanzando y evolucionando.

En ese sentido, conocer la historia se torna necesario, aún más si el espacio cobija el punto focal - llamado también el corazón- de una institución como es el caso de la Biblioteca de la Facultad de Enfermería de la Universidad Peruana Cayetano Heredia (UPCH), la cual es un elemento fundamental en la formación 
profesional en enfermería por ser un Centro de Recurso para el aprendizaje, docencia e investigación. Sin duda, la biblioteca, en el contexto de la educación superior, constituye un servicio clave para lograr las dos funciones que son la razón de ser de una universidad: la investigación o generación de conocimiento y la enseñanza o la comunicación de dichos conocimientos. Es así que el papel de la biblioteca en la calidad de la educación superior es primordial, pues constituye el principal apoyo académico en el suministro de información y conocimiento.

A continuación, conoceremos los antecedentes históricos de la Biblioteca de la Facultad de Enfermería de la Universidad Peruana Cayetano Heredia.

\section{ANTECEDENTES HISTÓRICOS}

Conocer los inicios de la biblioteca es, de cierta manera, remontarnos a una serie de hitos históricos de la Facultad de Enfermería, ya que ambas están estrechamente vinculadas. Por ello, estableceremos tres etapas significativas que ilustrarán el desarrollo de la institución bibliotecaria.

\section{Primera Etapa. La Escuela de Enfermeras Arzobispo Loayza y la creación de la biblioteca}

A fines del siglo XIX y comienzos del siglo XX, la Sociedad de Benericencia Pública de Lima SBPL tenía bajo su responsabilidad administrativa la mayoría de los hospitales ubicados en Lima, lo cual, a su vez, implicaba su preocupación en proporcionar a los pacientes una mejor atención que ayude a disminuir la incidencia de las enfermedades; para ello, era imprescindibie disponer de personal profesional preparado para brindar una mejor calidad de atención, siendo base primordial la formación de los profesionales en salud, específicamente en el campo de enfermería. Dicha necesidad se sustentaba en la experiencia exitosa en otros países, pues ya existían escuelas para la formación profesional de enfermeras.

La creación de la escuela surgió por iniciativa del Dr. Wenceslao Molina, inspector del Hospital Dos de Mayo, quien propone a la Junta General de Socios de la SBPL la creación de una Escuela de Enfermeros. La Junta aorueba la propuesta y el 18 de julio del 1915 comienza, inicialmente, a funcionar en el Hospital Dos de Mayo denominada, en ese entonces, Escuela Mixta de Enfermeros del Perú; luego, en 1917, funciona en el Hospital de Sanza Ana y, finalmente, en 1924, es trasladada a la parte central del Hospital Nacional Arzobispo Loayza por lo cual se la considera como una de las primeras escuelas de enfermeras del país.

En los años iniciales, la escuela funcionaba en el mismo Hospital Nacional Arzobispo Loayza, ya que durante esos años la escuela carecía de una infraestructura propia. Era evidente, entonces, la falta de instalaciones idóneas para albergar una biblioteca. Asimismo, en el campo de la ecucacion en enfermeria, la formación se desarrollaba sobre la base de un trabajo más hospitalario en el que la práctica era más importante que la teoría. Es decir, las 
estudiantes formaban parte del personal hospitalario, con una connotación de Escuela Hospitalaria. Así, lo afirma la profesora Margot Zárate, quien comenta que, en los albores del siglo XX, la enfermería estuvo más relacionada al desarrollo de los hospitales (medicina curativa) "evolucionando en este período de una actividad empirica." $L a$ institucionalización de la formación de las enfermeras se logra recién cuando surgen las primeras escuelas de enfermería en el Perú, como en el caso de la Escuela Mixta de Enfermeros (1915), luego denominada Escuela de Enfermeras Arzobispo Loayza, la que, actualmente, es la Facultad de Enfermería de la Universidad Peruana Cayetano Heredia.

En aquellos tiempos, el cuidado de los enfermos recaía también en las congregaciones religiosas de mujeres quienes cumplian una función social, espiritual y moral lo que significaba - como no podría ser de otra manera- que era de vital importancia su participación directa en la formación de enfermeras. Es así que, posteriormente, en 1922, la Escuela pasa a ser dirigida por la Congregación de Hijas de la Caridad de San Vicente de Paul, siendo la primera directora de la Escuela Sor Odila Broner y, luego, en 1924, la Rvda. Macire Sor Rosa Larrabure Correa. Justamente, durante dicha administración, el 19 de enero de 1931 y gracias a sus gestiones se consigue el terreno propio para la construcción del predio donde funcionaria la Escuela de Enfermeras Arzobispo Loayza, cuya donación fue realizada por el filántropo trujillano don Víctor Larco Herrera. El local está ubicado en el jirón Miguel Baquero Nro. 251, cel distrito de Lima con un área de $6,537.00 \mathrm{~m} 2$, local que hasta nuestros días funciona (ExEscuela de Enfermeras Arzobispo Loayza, hoy Facultad de Enfermería de la Universidad Peruana Cayetano Heredia). Este local fue construido en 1943 cuando era presidente de la República don Manuel Prado Ugarteche quien el 27 de julio del mismo año participó en la colocación de la primera piedra y comisionó a la Junta de Pro-Desocupados de Lima la ejecución de la obra. El 22 de julio de 1945 fue inaugurado el local que empieza a funcionar con cos niveles físicos y con una capacidad para 150 alumnos.

Según fuentes de información impresa, es a partir del período religioso cuando se crea la biblioteca. Para la profesora Fabiola Tavera (2004) "La. Biblioteca ha existido desde que las Hijas de la Caridad asumieron la conducción de la Escuela en $1924^{\prime 2}$ (sic). Dicho año admite confusión, pues según los documentos del Archivo de la Escuela de Enfermeras Arzobispo Loayza, fue en 1922 - año exacto- cuando la Congregación de Hijas de la Caridad de San Vicente de Paul asume la administración de la Escuela. Es decir, se había especulado que la Biblioteca nace con la Escuela. Sin embargo, la Escuela pudo contar recién con su Biblioteca en 1924; es decir, dos años después. Por tal razón, pocemos afirmar que fue en 1924 cuando la Biblioteca de Enfermería inició sus actividades, aproximadamente desde los comienzos de la gestión en la ExEscuela de Enfermeras Arzobispo Loayza, prestigiosa institución formaclora de

\footnotetext{
${ }^{1}$ Zarate M. Historia de la enfermería peruana. p. 53.

${ }^{2}$ Idem, p. 177.
} 
enfermeras profesionales con calidad y calidez humana y fue presidida por la directora la Rvda. Madre Sor Rosa Larrabure Correa quien pertenecía a la Congregación de Hijas de la Caridad de San Vicente de Paul.

Desde entonces, sus condiciones y capacidades de servicio han ido en aumento progresivo. La biblioteca, paulatinamente, se fue ampliando con abundante material hasta alcanzar hoy en día buenos resultados como la formación de una colección especializada en enfermería y ciencias afines. En un inicio, la biblioteca fue ubicada en el primer piso del local de la Ex-Escuela, primero en un ambiente limitado y con escaso material bibliográfico. Sin embargo, sus condiciones en el servicio se han ido incrementando. Esto debido a las donaciones de prestigiosas instituciones como la Fundación Kellogg, la Misión Económica de la Embajada de los Estados Unidos, CONCYTEC, Instituciones vinculadas a las ciencias de la salud, docentes y donaciones particulares. Durante esos años, la biblioteca ya contaba con una colección que ascendía a 2,500 titulos de material bibliográfico y estaba suscrita a 13 títulos de publicaciones periódicas. Evidentemente, no podemos dejar de observar que la biblioteca también padeció de algunos males comunes y perjudiciales como son: el reducido espacio, el poco presupuesto y el insuficiente recurso humano, io que generó que en algunos casos se viera limitado el desempeño en los servicios bibliotecarios.

Por otro lado, uno de los momentos más significativo en la historia de la Biblioteca de Enfermería fue en 1960 cuando asume la gestión Aurora Villanueva, primera bibliotecaria profesional responsable de la administración, quien estableció cambios favorables en el servicio. Dichos cambios iueron: ampliar el horario de atención, centrarse en un servicio más personalizacio y organizar el sistema de estantería abierta. En 1965, la bibliotecaria escribe el artículo "Nuestra Biblioteca" en la Revistas Ecos de Nuestra Escuela donde afirma "...la bibliotecaria ha dado más importancia al aspecto humano y de servicio personal... procurando atraer a las alumnas a la compañia de los libros y haciendo que la biblioteca sea el lugar a donde se dirija espontáneamente la estudiante..." .

Existen relatos testimoniales de ex-alumnas quienes recuerdan con nostälgia sus primeros pasos en la Biblioteca de Enfermería, pues se desarrollaban actividades creativas con el objetivo de despertar el interés de la lectura, estimular su capacidad de concentración y memoria. Es así, que se organizan exposiciones de libros, ferias de libros, concursos lúdicos de ajedrez y también se crea el "Premio al Mejor Lector". Asimismo, la Bibliotecaria menciona "...para estimular en las alumnas el interés por los libros se estableció un premio para las más asiduas lectoras, cuya entrega se verifica anualmente en la Semana de la Escuela...

${ }^{3}$ ECOS de Nuestra Escuela, ENE. Revista Anual de la Escuela Nacional de Enfermeras. p. 26.

${ }^{4}$ Op. cit, p. 27. 
Entre 1970 a 1972, asume la dirección de la Escuela Sor Pilar Caycho Vela, última religiosa de ese período, quien sintetiza el legado histórico impregnado de un espíritu cristiano y caritativo. Es decir, la influencia religiosa -que aún perdura- permitió delinear en las alumnas el espíritu filosófico cristianocatólico que significa la formación integral de la enfermera con valores éticos en atención al paciente. En 1973, las religiosas de las Hijas de la Caridad concluyeron su contrato con la SBPL, lo que implicaba también la renuncia a sus labores pedagógicas en la Escuela. Por lo tanto, desde 1973 asumen las funciones de la dirección de la escuela enfermeras civiles y ex-alumnas formadas dentro del espíritu de la congregación. Esto aseguraba la continuidad administrativa en el periodo de las hermanas; es decir, con la eficiencia y formación moral de sus antecesoras. Fue la enfermera civil Fabiola Tavera quien permaneció en el cargo durante doce años ininterrumpidos. Dentro de sus muchos aportes, y en relación a nuestro tema de interés, en 1974, realizó la ampliación de la infraestructura física y se construyó el tercer piso de la Escuela. Según menciona la profesora Fabiola Tavera (2004) "Se construyeron, en el tercer piso,...un ambiente grande para la Biblioteca (con un amplio mostrador para la atención), un cuarto para oficina y un cuarto para depósito de libros...",

En vista de ello, y como la institución disponía de nuevos ambientes fisicos, se incrementó el número de plazas para estudiantes en enfermería, por lo que era necesario incrementar también el número de recursos humanos en docentes y en personal administrativo. En el caso de la biblioteca, surge la figura de contratar a un bibliotecario, como así lo afirma la profesora Fabiola Tavera (2004): "Al contar con nuevos y amplios ambientes... la Dirección de la Escuela y la plana de profesoras, de acuerdo con la Sociedad de Beneficencia Pública de Lima, decidieron ampliar el número de plazas.... Fue necesario crear...dos pazas para personal auxiliar de disciplina, un bibliotecario..." . Sin embargo, no sabenos en realidad si dicha plaza fue ocupada y cuáles fueron sus aportes.

Segunda Etapa. La Facultad de Enfermería y la actividad bibiotecarta en el contexto universitario

A mediados de los años 60 , acorde con el avance científico y tecnológico ce la ciencia y por ende de la enfermería, la OPS/OMS sugería que la enferme ía en el Perú sea una profesión universitaria al igual que lo era en otros países. En realidad, desde muchos años antes, la Escuela había logrado una coincidencia de este enfoque, ya que era necesario en aquella época, como lo es hoy en cia, poseer una adecuada preparación. Es así que la Escuela vio favorable ele rar la formación profesional a un nivel universitario con el objetivo ce formar profesionales de la más alta calidad integral para el perfeccionamiento ce sus títulos académicos y por consiguiente, lograr el desarrollo de la enfermería como

\footnotetext{
${ }^{5}$ Tavera de Martínez, Fabiola. Historia de la Escuela Nacional de Enfermeres Arzobispo Loayza 1915-2003. p. 213.

${ }^{6}$ Ibídem
} 
disciplina profesional y científica. Se buscó entonces, adecuarse a las normas y finalidades de la Ley Universitaria Nro. 23733 aunque desde inicios de 1951 que ya se venían gestando los preliminares para lograr que la formación profesional en enfermería accediera a un estatus universitario.

Estos primeros intentos por lograr que se otorguen grados de bachiller y título universitario de licenciatura en enfermería se concretaron el 18 de diciembre de 1984 al firmarse el convenio académico entre la Universidad Peruana Cayetano Heredia y la Sociedad de Beneficencia Pública de Lima. El rector de la Universidad Peruana Cayetano Heredia era el Dr. Alberto Cazoria Talleri y el presidente del directorio de la SBPL era el Dr. Alejandro Souza Castañeda. Ambas instituciones firmaron el convenio académico por un períocio de cinco años para su total incorporación académica.

Una vez firmado el convenio, la Escuela de Enfermeras Arzobispo Loayza se incorpora académicamente a la Universidad Peruana Cayetano Heredia, -en un primer momento- a través de la Facultad de Medicina Alberto Hurtado. Ello marcó el inicio de lo que sería una nueva etapa de transformación y crecimiento en la profesión de enfermería. El convenio significaba que la mencionada universidad, debidamente acreditada, otorgaba los grados académicos universitarios a las estudiantes de enfermería de la Escuela de Enfermeras Arzobispo Loayza y asumía la responsabilidad en su formación profesional.

Un aspecto importante a resaltar es en la sexta cláusula del convenio de incorporación de la Escuela a la Universidad, firmado el 18 de dicienore de 1984, donde señala lo siguiente:

"...EL CONVENIO, que celebran,...LA UNIVERSIDAD PERLANA CAYETANO HEREDIA...Y LA SOCIEDAD DE BENEF.CENCIA PÚBLICA DE LIMA...

SEXTA.-Mientras se realizan los procesos acordados en el presente CONVENIO, la Universidad y la Escuela se comprometen progresivamente a:...

3) Coordinar el uso de sus Bibliotecas;..." "7.

Es decir, desde la época del convenio, la universidad veía con interés y dada su importancia el papel de la biblioteca en el entorno universitario y, con ello, se abría un nuevo período en la vida institucional.

Con el devenir del convenio, surgieron una serie de eventos afortunados que influyeron para el uso extensivo de la biblioteca. Entre estos estasa la investigación en salud comunitaria, el interés por parte de los docentes en continuar con su formación profesional traducido en obtener escudios de maestrías investigación previa para la titulación de las alumnas en enfemeria. En vista de ello, surgía un nuevo perfil profesional en enfermería con mayor competencia en investigación para lo cual la biblioteca tenía un papel

${ }^{7}$ Archivo de la Escuela de Enfermeras Arzobispo Loayza, 1984. 
preponderante. Por lo tanto, era indispensable responder a las necesiciacies de información. Como menciona la profesora Fabiola Tavera (2004:136) " Un aspecto que contribuyó al mejoramiento de la enseñanza en la Escuela fue el constante incremento de la Biblioteca."

Ya que la historia de la Biblioteca de Enfermería se encuentra adjunta a los orígenes de la ex-Escuela, mencionaremos que luego de una serie de impases, finalmente, el 15 de octubre de 1991, se suscribe un documento de incorporación de la Escuela de Enfermeras Arzobispo Loayza a la Universidad Peruana Cayetano Heredia, considerado como el segundo convenio. Una vez firmado dicho convenio la Escuela de Enfermeras se incorpora en forma definitiva a la Universidad Peruana Cayetano Heredia como unidad académica administrativa. Posteriormente, en sesión de la Asamblea Universitaria con fecha de 11 de febrero del 1998, se aprueba la creación de la Facultad de Enfermería de la Universidad Peruana Cayetano Heredia.

Para finalizar esta etapa, no podemos dejar de mencionar la participación del recurso humano en la Biblioteca de Enfermería, por ser el bibliotecario una pieza muy importante dentro de la Institución, ya que de este depence su funcionamiento para elevar el nivel de los procesos y de los servicios bibliotecarios. Asimismo, y después de la gestión iniciada por la bibliotecaria Aurora Villanueva durante la primera etapa en la Escuela de Enfermeras Arzobispo Loayza, el personal que trabajo en los años posteriores era insuficiente para los servicios que querían ofrecer y, además, sin formación académica en la profesión. Es decir, personal empírico. Según testimonio de las ex-alumnas, el personal que trabajo en la actividad bibliotecaria, sin tener la formación académica correspondiente, desarrolló con gran dedicación la tarea de la biblioteca. Asimismo, eran personas que hace muchos años que se desempeñaban en la biblioteca y habían adquirido con el tiempo cierta experiencia que mejoró su desempeño y los transformó en personal iclóneo para algunas tareas. Algunas de las principales razones de no disponer de personal profesional eran por la falta de exigencias para cubrir el cargo, ya cue estaba incluido como cargo técnico, por lo cual cualquier persona puede ingresar.

\section{Tercera Etapa. Situación actual de la Biblioteca de la Faculzed de Enfermería}

A lo largo de esta última década, la Biblioteca de Enfermería ha ido modîs uanco su estructura inicial, ampliando sus funciones y adaptándose a las nuevas tecnologías de la información; en suma, a la modernidad. Es así que el 06 de diciembre del 2004 fue inaugurada la remodelación de la Biblioteca de la Facultad de Enfermería de la Universidad Peruana Cayetano Herecia siendo decana la magister Margarita Alayo Sarmiento. La remodelación física incluyó la implementación del nuevo mobiliario con el equipamiento necesario, redistribución del espacio, cambio de estantería, equipos tecnológiccs y un espacio destinado a la sala de lectura. 
Acorde con el proceso de modernización, a partir del 2005, la Biblioteca de Enfermería cuenta con una bibliotecóloga profesional a cargo quien ejecuta funciones de gestión. Entre las diversas responsabilidades a realizar por dicho cargo se desarrolla un proceso de cambio orientado a una mayor especialización en los servicios de información con el objetivo de ofrecer un mejor servicio y por ende, contribuir a que la formación profesional en enfermería sea de calidad.

El 02 de agosto del mismo año, ya en otro período de gestión, y siendo decana la magister Margot Zarate León se aprueba en Consejo de Facultad el Reglamento de la Biblioteca de la Facultad de Enfermería en el que se definen sus runciones, se describe su organización, así como los servicios que proporciona, los derechos, los deberes de los usuarios y las responsabiiidades del personal.

De manera progresiva, se da inicio al proceso de automatización; de este moao, a mediados del 2005, y a través de la página web de la Biblioteca Central de la $\mathrm{UPCH}$, contamos con un catálogo en línea para la búsqueda y recuperacion de la información, cuya dirección electrónica es: http://www.upch.edu.pe/vrinve/dugic/bsqprueba/appbiblio/apppresiamo/catalogo s.htm

Hoy, su sede se encuentra en el tercer piso del local de la Faculad de Enfermería, sito en jirón Miguel Baquero 251-Lima (Espaida del Hospital Nacional Arzobispo Loayza). Asimismo, depende directamente de su Facultad, aunque trabaja en coordinación con la Biblioteca Central de la UPCH y, a su vez, forma parte del Sistema de Bibliotecas de la Universidad.

Al mismo tiempo, iniciamos la asesoría al cuerpo docente y somos parcicises en el desarrollo de la cultura informacional a partir de la temática en el uso ce la información, cuya labor se viene profundizando en las asignarurés de Investigación en Pregrado e Investigación Clínica en Posgrado. Ası. esta iniciativa se viene plasmando en los Talleres de capacitación: Estrategias de Búsqueda de información. Dicha tendencia capacita al estudiante para lograr el desarrollo de competencias informativas; es decir, desarrollar habilidades que le permitan al usuario acceder a la información y a los recursos de información de manera autónoma. Nuestra participación se realiza como docente invitado. según sílabo y, a partir de las invitaciones extendidas por las coordinadoras ce las asignaturas correspondientes. Actualmente, la Biblioteca de Enfermeria cispone de una colección que asciende a 4031 volúmenes entre libros, tesis, monog:arias y 32 títulos de revistas.

Los servicios que la Biblioteca brinda hoy en día son los siguientes:

* Consulta y referencia

* Lectura en sala

* Préstamo a domicilio

* Servicio de consulta en bases de datos bibliográficas

* Salas de estudio 


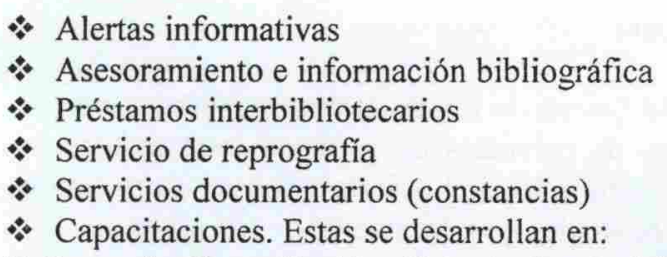

Talleres de Capacitación: Estrategias de búsqueda de información. Entre las principales bases de datos a texto completo tenemos: Access Medicine, elibro, e-ebrary, Harrison Online, UpToDate, ISI Web of Knowiedge, BestPractice, e-Libro, Cochrane, Annual Reviews, Scopus, Proquest, Finari, EBSCO, y las Bases de Datos de Libre Acceso (Biblioteca Virtual en Salud, Lilacs, Scielo y Medline, entre otros).

Capacitación para alumnos ingresantes. Brinda una orientación de casácter general a los alumnos ingresantes de enfermería sobre los productos y servicios que ofrece la Biblioteca.

DeCS: Pautas para su utilización. Palabras claves. Capacita al usuario en las técnicas de recuperación de la información, utilizando el DeCS, Descriptores en Ciencias de la Salud y contribuye en la selección de las palabras claves incluidas para el trabajo de las Tesis.

Referencias bibliográficas según normas Vancouver. Capacita al ustario en el manejo óptimo de las técnicas documentales para el registro y citado de las fuentes, lo cual contribuirá en la normalización de los criterios para la elaboración de las referencias bibliográficas en las tesis.

Hoy en día, la Biblioteca de Enfermería se encuentra trabajando en el proceso de autoevaluación con miras a la Acreditación Internacional del Programa de Pregrado de Enfermería de la UPCH. Tal es así que en su plan estratégico de la Biblioteca consideramos como uno de nuestros objetivos principa es la Acreditación Internacional de la Facultad. Nuestra biblioteca tiende a provectarse como Centro Gestor de Información y Productor de Conocimiento. Por lo tanto, no solo es el corazón, sino pronto o tal vez ya es el sistema circulatorio den io de la vida universitaria.

Finalmente, estimados lectores, hubiésemos querido disponer de más fientes para ilustrar los numerosos acontecimientos históricos sobre el pasado de nuestra Biblioteca de la Facultad de Enfermería de la Universidad Peruana Cayetano Heredia; sin embargo, esperamos haber contribuido con la recuperacion de nuestro pasado para la construcción del presente y futuro.

\section{Conclusiones}

$>$ El desarrollo histórico de la Biblioteca de la Facuitad de Enfermeria de la Universidad Peruana Cayetano Heredia se asocia a tres etapas significetivas: la primera, la creación de la Escuela de Enfermeras Arzobispo Loayza bajo la administración de la Congregación de Hijas de la Caridad de San Vicente de Paul; la segunda, la creación de la Facultad de Enfermería y su vinculación 
con el contexto universitario, y la tercera, la consolidación de la actividad bibliotecaria en la situación actual.

En el período de creación, la Biblioteca de Enfermería se caracterizo por su precariedad, debido a una serie de carencias en recursos económicos, en recursos humanos y en infraestructura, lo cual ocasionó que no se pudieron satisfacer adecuadamente las necesidades de información de los usuarios.

Los principales resultados obtenidos en la primera etapa del desertollo histórico de la Biblioteca de Enfermería se manifestaron en una labor muy meritoria que realizó la bibliotecaria Aurora Villanueva, pues planté una nueva concepción del trabajo bibliotecario; es decir, facilitó el desarro:lo de la biblioteca al implementar una nueva tendencia en la gestión bibliotecaria.

$>$ En la segunda etapa del desarrollo histórico de la Biblioteca de Enfemeria, se evidenciaron cambios positivos para el desarrollo del trabajo biblotecario al estimular su desarrollo ascendente, ya que la biblioteca fue el foco central en el nivel universitario.

En la última etapa del desarrollo histórico de la Biblioteca de Enfermería, se muestran cambios esenciales que permiten la modernización de la bibitoteca. Ello como consecuencia del nuevo escenario que responde a una estructura que integran servicios $y$ 'recursos bibliotecarios, tecnológicos e infraestructura, sistemas de información, e instalaciones con el fin de dar el debido soporte a las necesidades de docentes y de aprendizaje de la institución universitaria.

\section{BIBLIOGRAFIA}

1. ARCHIVO DE LA ESCUELA DE ENFERMERAS ARZOEISPO LOAYZA (1984).

2. ARCHIVO DE LA ESCUELA DE ENFERMERAS ARZCBISPO LOAYZA (1925).

3. ARCHIVO DE LA ESCUELA DE ENFERMERAS ARZCBISPO LOAYZA (1960-1984).

4. BASADRE, JORGE. (1970) Historia de la república del Perú. Lima: Editorial Universitaria.

5. CABANILLAS TORRES, Mariella Isabel; RODRIGUEZ BENDEZÚ, Yolanda. (1993) Historia de la Escuela de Enfermeras Arzobispo Loayza en el contexto del desarrollo de la enfermeria peruana. Tesis de Licenciatura. Lima: Universidad Peruana Cayetano Heredia.

6. DOMÍNGUEZ AROCA, María Isabel. (2005) La bibloteca universitaria ante el nuevo modelo de aprendizaje: docences y bibliotecarios, aprendamos juntos porque trabajamos jumios. En: RED. 
Revista de Educación a Distancia. Disponiblé en: http://www.um.es/ead/red/M4/dominguez9.pdf

7. Entrevista a la Docente ZARATE LEÓN, Margot. Octubre 2010

8. Entrevista a la Docente SALAZAR GARCíA, María del Carmen. Octubre 2010.

9. PADILLA LÓPEZ, Elizabeth Madelaine. (1998) Actividades tradicionales de la Escuela Nacional de Enfermeras Arzobispo Loayza. Tesis de Licenciatura. Lima: Universidad Peruana Cayetano Heredia.

10. PAMO REYNA, Oscar Guillermo. (2007) Una visión nistórica de la participación femenina en la profesión médica. En: Revistà ce la Sociedad Peruana de Medicina Interna; 20 (3). Disponible en: http://sisbib.unmsm.edu.pe/bVrevistas/spmi/v20n3/pdf/a06v20n3.pof

11. PERÚ. Ley Universitaria $\mathrm{N}^{\circ}$ 23733. Disponiblê en: http://www.unmsm.edu.pe/ogp/ARCHIVOS/LEY

UNIVERSITARIA.htm

12. TAVERA DE MARTÍNEZ, Fabiola. (2004) Historia de la Escuela Nacional de Enfermeras Arzobispo Loayza 1915-2003. Lima: UPCH, Facultad de Enfermería.

13. UNIVERSIDAD PERUANA CAYETANO HEREDIA (2001). Escuela de Postgrado Víctor Alzamora Castro. Diálogos universitavios de postgrado. v.1. Rol social de la universidad y de los postgrados. Lima: UPCH. Escuela de Postgrado Víctor Alzamora Castro.

14. VERDERESE, O. (1979) Análisis de la enfermería en Américá Latina, pp.315-316. En: Educación médica salud. 13.

15. VILLANUEVA, Aurora. (1965) Nuestra Biblioteca. ECOS de Nuestra Escuela, ENE, pp.26-27. Revista Anual de la Escuela Naciona! de Enfermeras; 1(1) Diciembre.

16. ZARATE LEÓN, Margot. (1992) Historia de la enfermería peruana. pp. 51-58. Revista Horizonte de Enfermeria; 3(2).

\section{Agradecimientos $^{8}$}

${ }^{8}$ Deseo aprovechar estas últimas líneas para hacer un merecido reconocimiento a las profesoras Mg. Margot Zarate León y a la Lic. María del Carmen Salazar García, por el apoyo en el relato testimonial. Gracias por su tiempo y paciencia, y por compartir su interés por nuestro pasado institucional. 